\title{
Comparative Analysis of Variants of Token Bucket Traffic Meter Algorithms for QoS Router using UDP as Traffic Agent
}

\author{
${ }^{1}$ Oyetunji M.O., ${ }^{2}$ Emuoyinbofarhe O.J., ${ }^{3}$ Oladosu J.B, ${ }^{4}$ Oladeji F.O. \\ ${ }^{I}$ Department of Computer Science and Mathematics, MTU, Lagos/Ibadan Express Way, State . \\ ${ }^{2}$ Department of Computer Science and Engineering, LAUTECH, Ogbomoso. \\ ${ }^{3}$ Department of Computer Science and Engineering, LAUTECH, Ogbomoso \\ ${ }^{4}$ Department of Computer Science, University of Lagos, Akoka, Lagos.
}

\begin{abstract}
:
Traffic meter algorithms serve as a means of examining traffic stream's conformance with service level agreement between customers (traffic sources) and Internet Service provider at the edge router of a differentiated service domain for proper quality of service admission control. This paper presented comparative analysis of variants of token bucket meter algorithms for QoS router using user datagram protocol (UDP) as traffic agents and exponential ON/OFF as traffic generator. The research adopted simulation technique to carry out the design of network models or topologies using the same parameter setting to implement the algorithm of token bucket variants of traffic meter. The following metrics were used for the evaluation: throughput, fairness rate, loss rate and one-way packet delay. The evaluated results were ranked and further subjected to 2-way analysis of variance (ANOVA) model to indicate the significant differences among the traffic meter algorithms. Based on ranking system, TRTCM was ranked first in terms of throughput (with 67117) and fairness rate (with 0.2586) and TBM was ranked first in terms of loss rate (with 74.003) and one-way packet delay (with 0.09304). The 2-way ANOVA model showed the significant differences among the traffic meter algorithms considered for the simulation.
\end{abstract}

Keywords: TRTCM, SRTCM, TBM, UDP, TCP and Traffic meter

\section{INTRODUCTION}

The need to improve on the deployments of multimedia applications over the Transport Control Protocol/Internet protocol (TCP/IP) suite is of great important because it requires very high bandwidth, bursty data transmission and stringent delay constraints for quality of service (QoS) assurance (Oyetunji, Oladeji, \& Emuoyinbofarhe, 2012; Pandit, 2006) The TCP/IP protocol suites define set of rules that communicating devices must follow to communicate with one another over the Internet (Ferouzan, 2013).

In attempt to work on the QoS of the conventional internet, IETF came up with two architectures: integrated services (IntServ) and differentiated services (DiffServ) architecture. The IntServ architecture is characterized by resource reservation for each session or flow by the router along the path of the traffic to the destination. Its problem is scalability when thousands of applications are requesting for reservation at the same time. This limitation of IntServ architecture brought about the development of DiffServ architecture which makes provision for traffic flows demanding same treatment from the network to be aggregated at the routers (Braden, Clark, \& Shenker, 1994; Clark \& Fang, 1998; Kurose \& Ross, 2000).

DiffServ is based on a simple model which attempts to move complexity of the network to the edge router and keep the functionality of the core network as simple as possible (Blake et al., 1998). Core devices perform only the forwarding operation (Lochin \& Anelli, 2009). The admission control mechanism or conditioner is introduced at the boundary of the DiffServ network or domain to check whether a service request is to be granted or rejected 
(Georgoulas, 2007). The DiffServ model uses this mechanism at the network edge (edge routine) to measure, mark, shapen or police packets if necessary.

Before traffic flows enter the network core from different edge domains, they need to be marked either as in-profile or out-of-profile at their respective edge networks. Traffic meter algorithms measure the properties of the stream of packets that arrive to the router against a set traffic profile. It guides in regulating the injection of traffic to the network (Nordström, 2006). This paper considers comparative analysis of token bucket variants of traffic meters algorithms: token bucket meter (TBM), single rate three color meter (SRTCM) and two rate three color meter (TRTCM) algorithms using user datagram protocol as traffic agent because most multimedia applications are deployed on UDP.

\section{TRAFFIC CLASIFICATION AND CONDITIONING}

Traffic conditioning normally takes place at the boundary of a network. Differentiated services are then extended across a differentiated service domain (DSD) boundary by establishing a Service Level Agreement (SLA) which describes the type of service to be provided from a service provider to a customer between upstream network and downstream DSD. The SLA guides in specifying the traffic classification and re-marking rules, and it may also specify traffic profiles and action to traffic stream which can be in-profile (traffic stream that complies with SLA) or out-of-profile (traffic stream that do not comply with SLA). Traffic Conditioning Agreement (TCA) is derived from this Service Level Agreement. (Blake et al., 1998; Carlson et al., 1998; Oyetunji et al., 2012; Strauss, Kourie, \& Olivier, 2005).

From the TCA we can further define the role of traffic classification. It identifies a subset of traffic that will receive a differentiated service such that the identified traffic stream will then be conditioned and/or mapped to one or more behaviour aggregates. Traffic conditioning performs metering, shaping, policing and marking or re-marking of traffic to ensure that traffic entering a domain conforms to the rules specified in the TCA as shown in Figure 1 (Miller, 2009). In the simplest model, each packet is either in-profile or out-of-profile based on the metering result at the arrival time of the packet. In-profile packets obtain better traffic conditioning and forwarding treatment than outof-profile packets (Blake et al., 1998; Strauss et al, 2005).

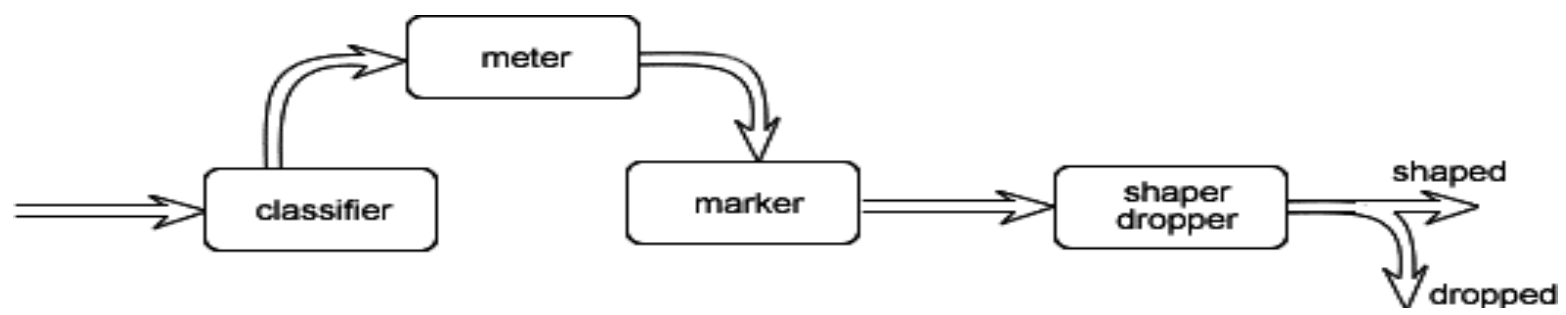

Figure 1: DiffServ traffic conditioner block in the edge router Source: (Andreozzi, 2000)

\section{VARIANTS OF TOKEN BUCKET TRAFFIC METER ALGORITHMS}

The variants of token bucket traffic meter algorithms considered in this paper are token bucket meter (TBM) algorithm, single rate three color meter (SRTCM) algorithm and two rate three color meter (TRTCM) algorithm.

\section{Token Bucket Meter (TBM) Algorithm}

TBM measures traffic stream based on two traffic conditioning parameters: Committed Information Rate (CIR) and Committed Burst Size (CBS) with 2 drop precedence (Complaint and Non-Compliant) (Freed, Amara, \& Borella, 2006). The meter is identified in form of token bucket (C). The maximum size of C is CBS. Originally, the token bucket is full, i.e. $T_{C}(0)=C B S$, where $T_{C}$ is the token count. If $\left(T_{C}<C B S\right)$ then $T_{C} \leftarrow T_{C}+1$ else $T_{C}$ is not incremented (Freed, Amara, \& Borella, 2006).

The marker makes use of meter to determine the color of the newly arrived packets. Assume a packet of size B bytes arrives at time $t$. If $T_{C}(t)-B \geq 0$, then the packet is marked green and $T_{C} \leftarrow T_{C}-B$. Else the packet is marked red and $\mathrm{T}_{\mathrm{C}}$ remain unchanged as shown in Figure 2 (Freed et al., 2006). 


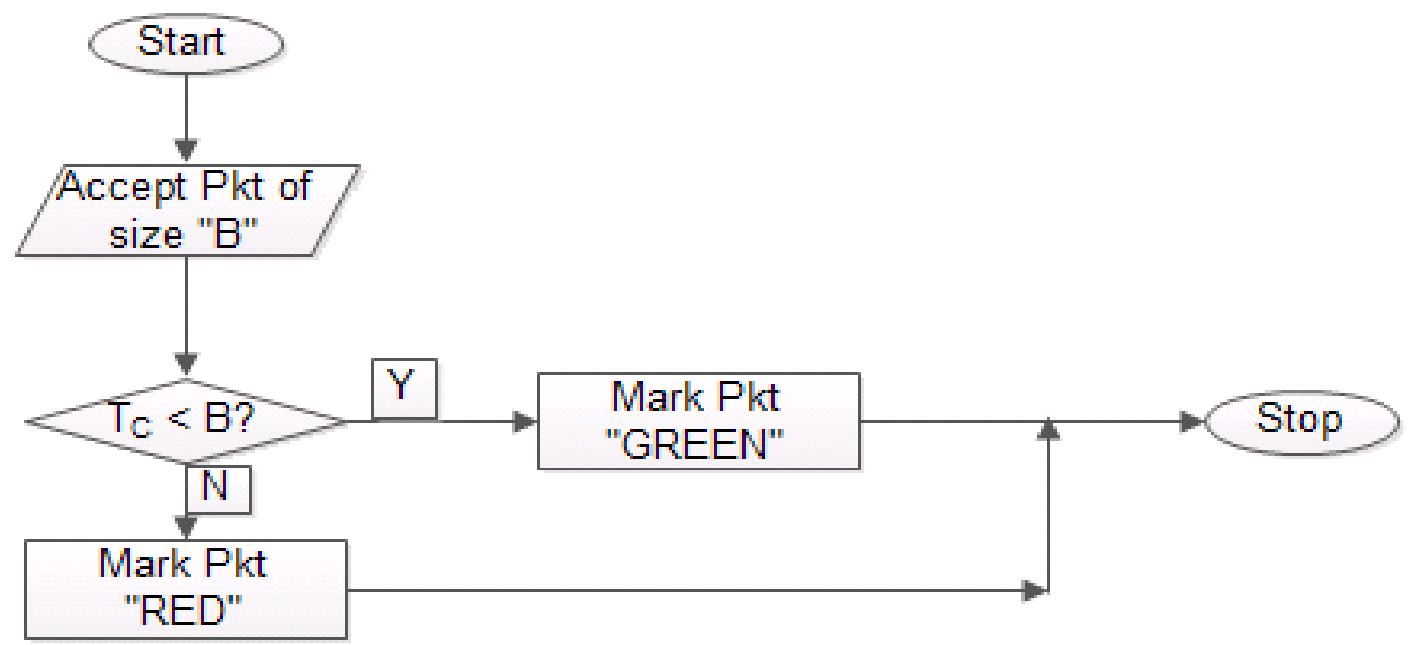

Figure 2: Flow Chart for TBM algorithms

\section{Single Rate Three Color Meter (SRTCM) Algorithm}

The SRTCM measures traffic stream based on three parameters: CIR, CBS and EBS coupled with three drop predecences (one for complaint packets and two for non-compliant packets) and passes the measurements to its marker (Freed et al., 2006). SRTCM uses single rate and it is identified in terms of two buckets: C and E. The capacity of $\mathrm{C}$ is CBS and that of $\mathrm{E}$ is EBS. Originally, both tokens are full, i.e. $T_{C}(0)=C B S$ and $T_{E}(0)=E B S$.

If $\left(\mathrm{T}_{\mathrm{C}}<\mathrm{CBS}\right)$ then $\mathrm{T}_{\mathrm{C}} \leftarrow \mathrm{T}_{\mathrm{C}}+1$ else

If $\left(T_{E}<\right.$ EBS) then $T_{E} \leftarrow T_{E}+1$ else Neither $T_{C}$ nor $T_{E}$ are incremented (Freed, Amara, \& Borella, 2006) When a packet of size $\mathrm{B}$ arriving at time $t$, the following happens:

If $T_{C}(t)-B \geq 0$, the packet is marked green and then $T_{C} \leftarrow T_{C}-B$

If $T_{E}(t)-B \geq 0$, the packet is marked yellow and then $T_{E} \leftarrow T_{C}+1$

If neither of the previous cases is valid, the packet is marked red. $\mathrm{T}_{\mathrm{C}}$ and $\mathrm{T}_{\mathrm{E}}$ remain unchanged as shown in Figure 3

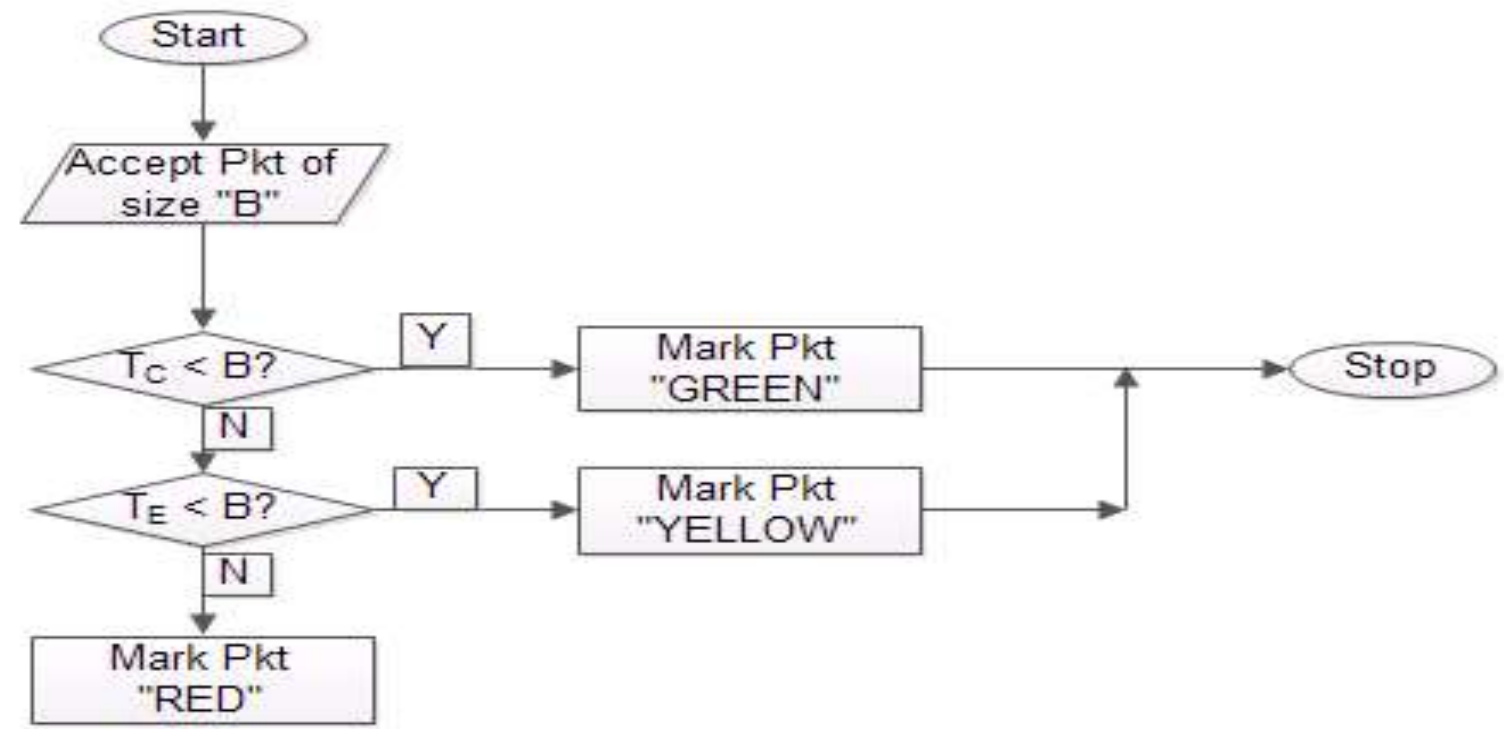

Figure 3: Flow Chart for SRTCM algorithms 


\section{Two Rate Three Color Meter (TRTCM) Algorithm}

The TRTCM measures traffic stream based on four traffic conditioning parameters: Committed Information Rate (CIR), Peak Information Rate (PIR) and two burst sizes namely Committed Burst Size (CBS) and Peak Burst Size (PBS) with 3 drop precedence (one complaint and two non-compliant packets). TRTCM uses two rates (CIR and PIR) and it is also identified in terms of two buckets: $\mathrm{C}$ and P. The capacity of C is CBS and that of $\mathrm{P}$ is PBS. For clearity sake, its algorithm is discussed in (Heinanen \& Guérin, 1999). Originally, both token buckets are full, i.e. $\operatorname{Tc}(0)=\mathrm{CBS}$ and $\mathrm{Tp}(0)=$ PBS.

* If $(\mathrm{Tc}<\mathrm{CBS})$ then $\mathrm{Tc} \leftarrow \mathrm{Tc}+1$ else

* If $(\mathrm{TP}<\mathrm{PBS})$ then $\mathrm{TP}<\mathrm{T}_{\mathrm{P}}+1$ else neither Tc nor $\mathrm{T}_{\mathrm{P}}$ are incremented

When a packet of size $\mathrm{B}$ arrives at time $\mathrm{t}$, the following happen:

* If $\mathbf{T c}(\mathbf{t})-\mathbf{B} \geq \mathbf{0}$, the packet is marked as green and $\mathbf{T C} \leftarrow \mathbf{T C}-\mathrm{B}$

* If $\mathbf{T c}(\mathbf{t})-\mathbf{B}<\mathbf{0}$ and $\mathbf{T p}(\mathbf{t})-\mathbf{B} \geq \mathbf{0}$, the packet is marked yellow and $\mathbf{T}_{\mathrm{P}} \leftarrow \mathbf{T}_{\mathrm{P}}+1$

If neither of the previous cases is valid, the packet is marked red. $\mathbf{T}_{\mathbf{C}}$ and $\mathbf{T}_{\mathbf{P}}$ remain unchanged as shown in Figure 4 (Freed et al., 2006).

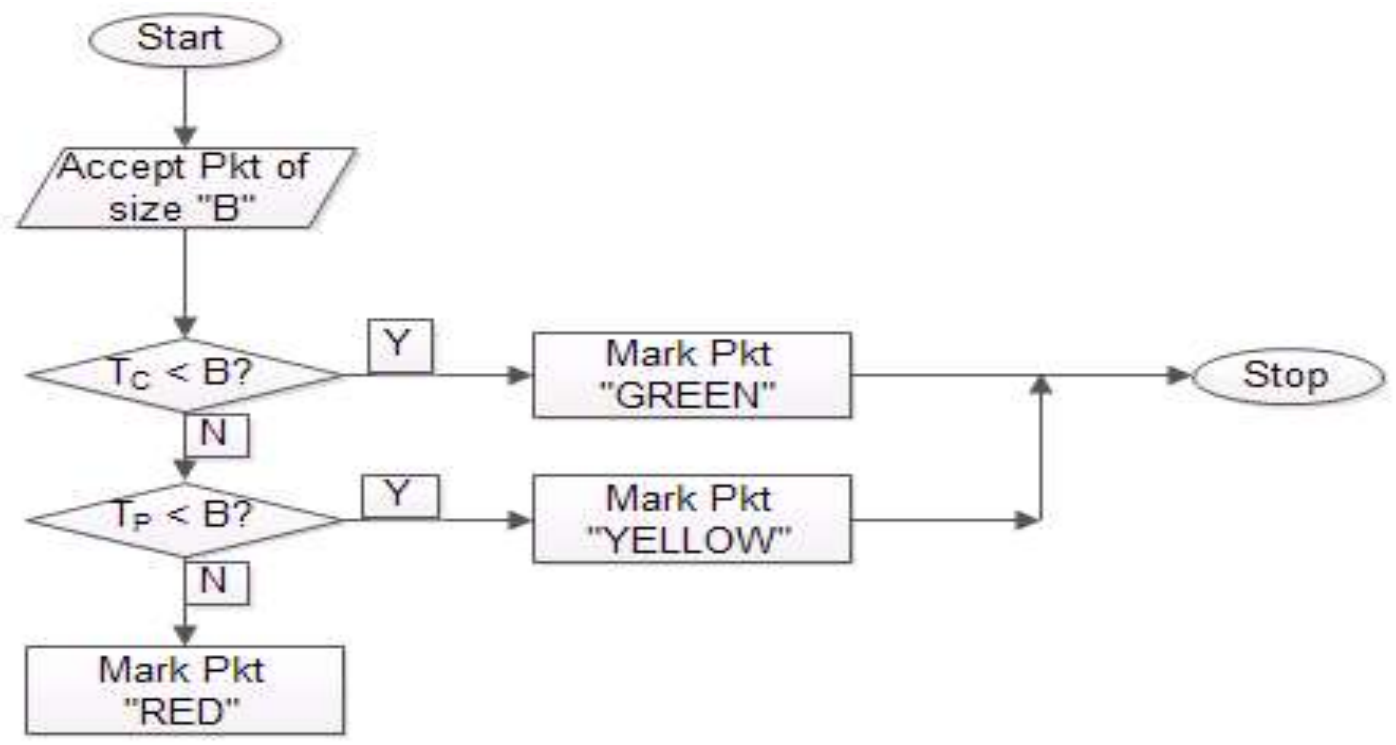

Figure 4: Flow Chart for TRTCM algorithms

\section{METHODOLOGY}

This research adopts simulation approach to carry out design and simulation of network topology to implement token buckets variants of traffic meter algorithms in order to create platform for absolute comparison among the meter algorithms on UDP as traffic agents using exponential ON/OFF as traffic generator as shown in Figure 5. The topology was simulated using a software simulator called network simulator-2 (ns-2).

The data generated from the simulation experiments were traced into files, analyzed and evaluated based on the following network performance metrics: throughput, fairness rate, loss rate and one-way packet delay to showcase the strengths and weaknesses of the meter algorithms. The results of the evaluation based on the performance metrics were further analyzed using two-way analysis of variance to show the significance differences among the studied meter algorithms.

\section{EXPERIMENTAL SETUP}

Since multimedia applications are expected to be routed through a core router, the research used user datagram protocol (UDP) as traffic agents that requires no acknowledgement. Each source is conditioned differently 
with its own parameter settings. Exponential ON/OFF was used to generate traffic from the eight sources as shown in Figure 2. Buffer size is assumed to be finite and the server is the bottlenecked core router with a deterministic capacity (bandwidth).

\section{SIMULATION TOPOLOGY}

The network topology design shown in Figure 5 represents the network models used for this research to implement the algorithms of the variants of token bucket traffic meters (token bucket meter (TBM), single rate three color meter (SRTCM) and two rate three color meter (TRTCM)). In the network topology, the same parameter settings were used to create platform for comparison among the traffic meter algorithms. The packet sizes for the simulated experiments were varied using 500bytes, 1000bytes and 2000bytes for the first, second and third scenarios respectively for each traffic meter algorithm to run the simulation three times.

The network topologies were designed to consist of 21 nodes (eight nodes are for sources, three nodes are for edge routers, two nodes are core routers and the remaining eight nodes are for destinations). The node-to-node network links from sources to destinations were configured with bandwidth of $10 \mathrm{Mbps}$ and link delays of $5 \mathrm{~ms}$ except from the core router $\mathrm{C} 2$ to edge router E3 which was set as 5Mbps bandwidth and $5 \mathrm{~ms}$ link delay as described in Figure 5. The core to core router configuration was set to 5Mbps intentionally to study the effect of congestions at the core routers. The sources $\left(\mathrm{S}_{1}, \mathrm{~S}_{2}, \mathrm{~S}_{3}, \mathrm{~S}_{4}\right)$ generated traffic streams with Exponential ON/OFF using UDP as traffic agent and send them to ingress edge router (E1).

Likewise, the sources $\left(\mathrm{S}_{5}, \mathrm{~S}_{6}, \mathrm{~S}_{7}, \mathrm{~S}_{8}\right)$ also generated traffic streams via the same medium using UDP as traffic agent and send them to edge router (E2). At the ingress routers (E1 and E2), the incoming traffic streams are admitted, classified and conditioned with traffic conditioning functions such as metering, marking and policing using associated differentiated services code point (DSCP) to carry out traffic profiles enforcement before sending them to the core router for forwarding. Each meter algorithm measured the traffic stream to ensure whether the traffic stream is in-profile or out-of-profile for proper traffic admission control. The core router buffers the packets into respective queues using priority scheduling discipline to forward them to respective destinations $\left(\mathrm{D}_{1}, \mathrm{D}_{2}, \mathrm{D}_{3}\right.$, $\mathrm{D}_{4}, \mathrm{D}_{5}, \mathrm{D}_{6}, \mathrm{D}_{7}$ and $\mathrm{D}_{8}$ ) through the egress router (E3). The transmission mode used between sources and destinations were full duplex transmission modes while simplex transmission mode was used between edge router and core router and also between core router and core router.

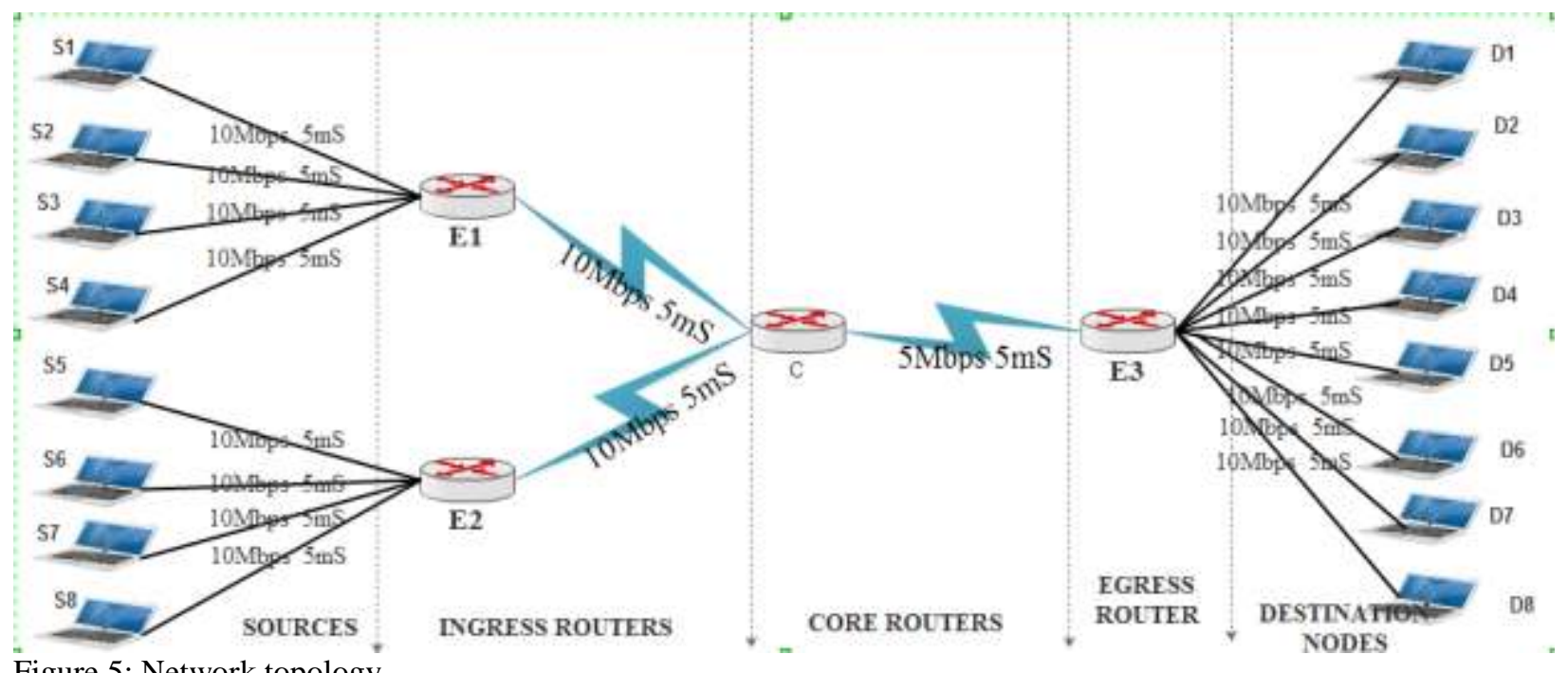

Figure 5: Network topology

\section{RESULTS}

The simulations were carried out for 80 seconds and the data generated were traced into an output file for performance metrics analyses. The analyses of the results were based on the following metrics: throughput, fairness rate, loss rate and one-way packet delay. The analyzed results were further subjected to ranking and two-way 
ANOVA model using two factors: traffic meter type (TRTCM, SRTCM and TBM) and packet sizes (500B, 1000B and 2000B). Two hypotheses were setup: Null hypothesis (shows no significance difference) and alternative hypothesis (shows significance differences among the traffic meters). $\alpha$ is taken to be 0.05 and the calculated Pvalue is compared with $\alpha$ to know if there is any significant difference or not. If P-value $<\alpha$, there is significant difference else there is no significant difference.

\section{a. Analysis Based on Throughput}

The higher the throughput value, the better the performance of the meter algorithm. Throughput is taken as the work done or total number of packets that gets to the destination. Comparing TRTCM, SRTCM and TBM in terms of throughput, Table 1 shows the throughput values of token bucket variants of traffic meter algorithms: TRTCM, SRTCM and TBM algorithms for 80 seconds simulation time interval each. The result gotten for each traffic meter in Table 1 was showcased graphically with bar chart in Figure 6. Figure 7 showed the 2-way ANOVA analyses with p-value of 0.000 which makes the null hypothesis to be rejected for alternative hypothesis. It shows that there is significant difference among the traffic meters. From the analyses, TRTCM algorithm was ranked first, followed by SRTCM and then TBM. Applications that require high throughput value on UDP traffic agent could make use of TRTCM algorithm.

Table 1: Throughput analyses for the traffic meters

\begin{tabular}{llll}
\hline & \multicolumn{3}{c}{ Traffic Meters } \\
\cline { 2 - 4 } \multicolumn{1}{c}{ SCENERIOS } & TRTCM- & SRTCM- & TBM- \\
$\mathbf{1}^{\text {st }}(\mathbf{5 0 0}$ Bytes $)$ & UDP & UDP & UDP \\
$\mathbf{2}^{\text {nd }}(\mathbf{1 0 0 0}$ Bytes) & 500432 & 100285 & 100009 \\
$\mathbf{3}^{\text {rd }}(\mathbf{2 0 0 0}$ Bytes) & 50462 & 50252 & 50024 \\
Avg. & 50311 & 50019 \\
Throughput & $\mathbf{6 7 1 1 7}$ & $\mathbf{6 6 9 4 9}$ & $\mathbf{6 6 6 8 4}$ \\
Rank & $\mathbf{1}^{\text {st }}$ & $\mathbf{2}^{\text {nd }}$ & $\mathbf{3}^{\text {rd }}$ \\
\cline { 2 - 4 } & & & \\
\end{tabular}

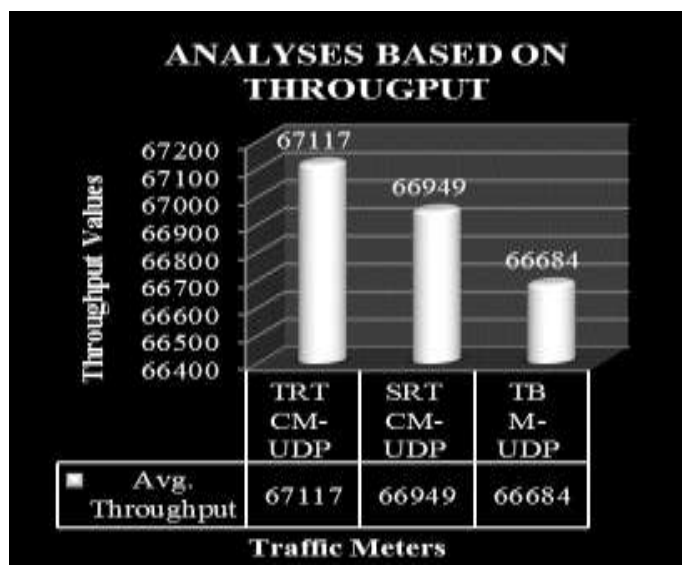

Figure 6: Throughput analyses for the traffic meters 


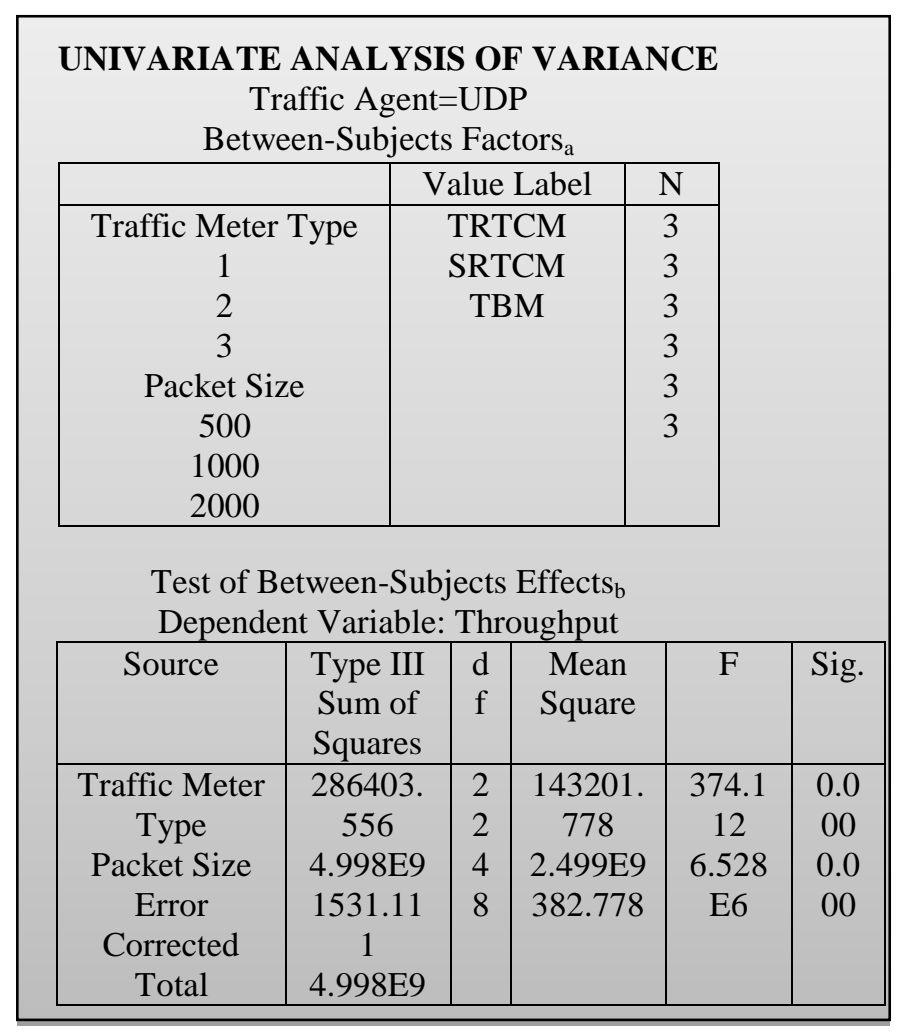

Homogeneous Subsets Throughput Duncan

\begin{tabular}{|c|c|c|c|c|}
\hline \multirow[b]{2}{*}{$\begin{array}{c}\text { Traffic Meter } \\
\text { Type }\end{array}$} & \multirow[b]{2}{*}{$\mathrm{N}$} & \multicolumn{3}{|c|}{ Subset } \\
\hline & & 1 & 2 & 3 \\
\hline TBM & 3 & $6.67 \mathrm{E} 4$ & & \\
\hline SRTCM & 3 & & $6.69 \mathrm{E} 4$ & \\
\hline $\begin{array}{c}\text { TRTCM } \\
\text { Sig. }\end{array}$ & 3 & 1.000 & 1.000 & $\begin{array}{c}6.71 \mathrm{E} 4 \\
1.000\end{array}$ \\
\hline
\end{tabular}

Figure 7: ANOVA analyses for throughput using UDP as traffic agent

\section{b. Analysis Based on Fairness Rate}

The higher the fairness rate value, the better the performance of the traffic meter. Fairness rate is evaluated based on Jain fairness index (Jain, 1990) which states that

Jain index $\left(\mathrm{I}_{\text {Jain }}\right)=\frac{\left(\sum_{i=1}^{n} x_{i}\right)^{2}}{n \sum_{i=1}^{n} x_{i}{ }^{2}}$

Where $\mathrm{n}$ is the number of active queues and $\mathrm{x}_{\mathrm{i}}$ is the number of packets that were transmitted in queue $\mathrm{i}$. Comparing TRTCM, SRTCM and TBM in terms of fairness rate, Table 2 shows that TRTCM algorithm was ranked first, followed by SRTCM and then TBM. The result gotten for each traffic meter in Table 2 was represented graphically with bar chart in Figure 8. Figure 9 showed the 2-way ANOVA analyses with p-value of 0.000 which makes the null hypothesis to be rejected for alternative hypothesis. It shows that there is significant difference among the traffic meters. Applications that require high fairness rate value on UDP traffic agent could make use of TRTCM algorithm 
Table 2: Fairness rate analyses for the traffic meters

\begin{tabular}{|c|c|l|c|}
\hline \multirow{2}{*}{ SCENERIOS } & \multicolumn{3}{|c|}{ Traffic Meters } \\
\cline { 2 - 4 } & $\begin{array}{l}\text { TRTCM- } \\
\text { UDP }\end{array}$ & $\begin{array}{c}\text { SRTCM- } \\
\text { UDP }\end{array}$ & TBM-UDP \\
\hline 1st (500Bytes) & 0.2494 & 0.1267 & 0.12531 \\
\hline $\begin{array}{c}\text { 2nd } \\
\text { (1000Bytes) }\end{array}$ & 0.2621 & 0.1285 & 0.12579 \\
\hline $\begin{array}{c}\text { 3rd } \\
\text { (2000Bytes) }\end{array}$ & 0.2643 & 0.1285 & 0.12573 \\
\hline Avg. Fairness & $\mathbf{0 . 2 5 8 6}$ & $\mathbf{0 . 1 2 7 9}$ & $\mathbf{0 . 1 2 5 6}$ \\
\hline Rank & $\mathbf{1}^{\text {st }}$ & $\mathbf{2}^{\text {nd }}$ & $\mathbf{3}^{\text {rd }}$ \\
\hline
\end{tabular}

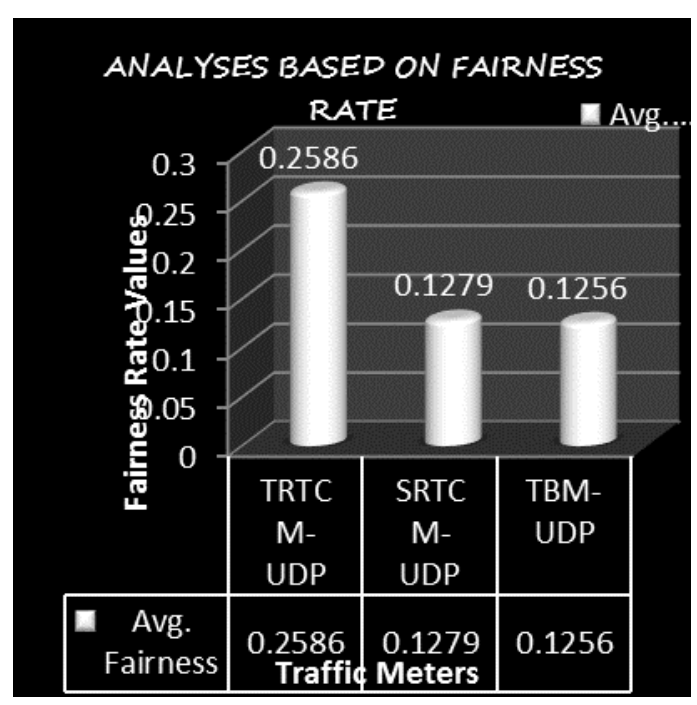

Figure 8: Fairness rate analyses for the traffic meters 
a. UNIVARIATE ANALYSIS OF VARIANCE

Traffic Agent=UDP

Between-Subjects Factors

\begin{tabular}{|c|c|c|}
\hline & $\begin{array}{l}\text { Value } \\
\text { Label }\end{array}$ & $\mathrm{N}$ \\
\hline Traffic Meter Type & TRTCM & 3 \\
\hline 1 & SRTCM & 3 \\
\hline 2 & TBM & 3 \\
\hline 3 & & 3 \\
\hline $\begin{array}{l}\text { Packet Size } \\
500\end{array}$ & & $\begin{array}{l}3 \\
3\end{array}$ \\
\hline 1000 & & \\
\hline 2000 & & \\
\hline
\end{tabular}

Test of Between-Subjects Effects Dependent Variable: Fairness Rate

\begin{tabular}{|l|l|l|l|l|l|}
\hline Source & $\begin{array}{c}\text { Type III } \\
\text { Sum of } \\
\text { Squares }\end{array}$ & df & $\begin{array}{l}\text { Mean } \\
\text { Squar } \\
\text { e }\end{array}$ & F & Sig. \\
\hline Traffic Meter & 47.757 & 2 & & & \\
Type & 0.578 & 2 & 173.8 & 942.0 & 0.0 \\
Packet Size & 0.738 & 4 & 78 & 15 & 00 \\
Error & 49.073 & 8 & 0.289 & & \\
orrected Total & & & 0.185 & 1.566 & 0.3 \\
& & & & & 15 \\
\hline
\end{tabular}

Homogeneous Subsets

Fairness rate

Duncan

\begin{tabular}{|l|c|l|c|}
\hline \multirow{2}{*}{$\begin{array}{l}\text { Traffic Meter } \\
\text { Type }\end{array}$} & \multirow{2}{*}{$\mathrm{N}$} & \multicolumn{2}{|c|}{ Subset } \\
\cline { 3 - 4 } & & & 2 \\
\hline TBM & 3 & 0.12560 & \\
SRTCM & 3 & 0.12792 & \\
TRTCM & 3 & & 0.25861 \\
Sig. & & 0.545 & 1.000 \\
\hline
\end{tabular}

Figure 9: ANOVA analyses for fairness rate using UDP as traffic agent

\section{c. Analysis Based on Loss Rate}

The lower the loss rate value, the better the performance of the traffic meter algorithm. Loss rate is evaluated based on the formula stated below

\section{Loss Rate $=\frac{(\text { Packet enqued-packet received })}{\text { packet enqued }} * 100$}

Where packet enqued are the packets that are queued up at the ingress routers and packet received is the total packet that got to the destination. 
Comparing TRTCM, SRTCM and TBM in terms of loss rate, Table 3 shows that TBM algorithm was ranked first, followed by SRTCM and then TRTCM. The result gotten for each traffic meter in Table 3 was represented graphically with bar chart in Figure 10. Figure 11 showed the 2-way ANOVA analyses with p-value of 0.002 which makes the null hypothesis to be rejected for alternative hypothesis. It shows that there is significant difference among the traffic meters. Applications that require low loss rate value on UDP traffic agent could make use of TBM algorithm.

Table 3: Loss rate analyses for the traffic meters

\begin{tabular}{|l|c|c|c|}
\hline \multirow{2}{*}{ SCENERIO } & \multicolumn{3}{|c|}{ TRAFFIC METERS } \\
\cline { 2 - 4 } & TRTCM-UDP & SRTCM-UDP & TBM-UDP \\
\hline 1st (500Bytes) & 74.61 & 73.84 & 73.93 \\
\hline 2nd (1000Bytes) & 74.7 & 74.27 & 74.05 \\
\hline 3rd (2000Bytes) & 74.7 & 74.19 & 74.03 \\
\hline Avg. Loss Rate & $\mathbf{7 4 . 6 7}$ & $\mathbf{7 4 . 1}$ & $\mathbf{7 4 . 0 0 3}^{\text {id }}$ \\
\hline Ranks & $\mathbf{3}^{\text {rd }}$ & $\mathbf{2}^{\text {nd }}$ & $\mathbf{1}^{\text {st }}$ \\
\hline
\end{tabular}

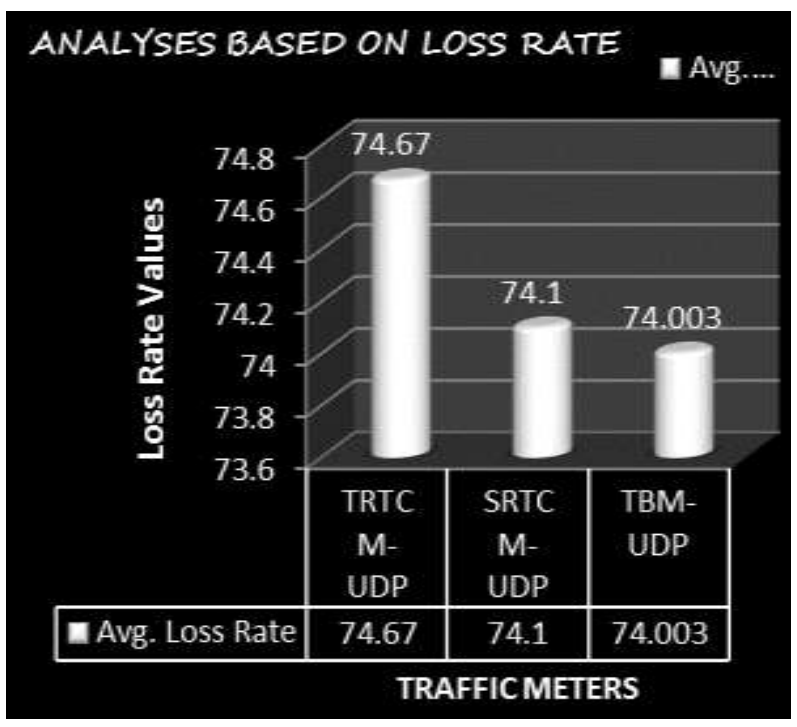

Figure 10: Loss rate analyses for the traffic meters 


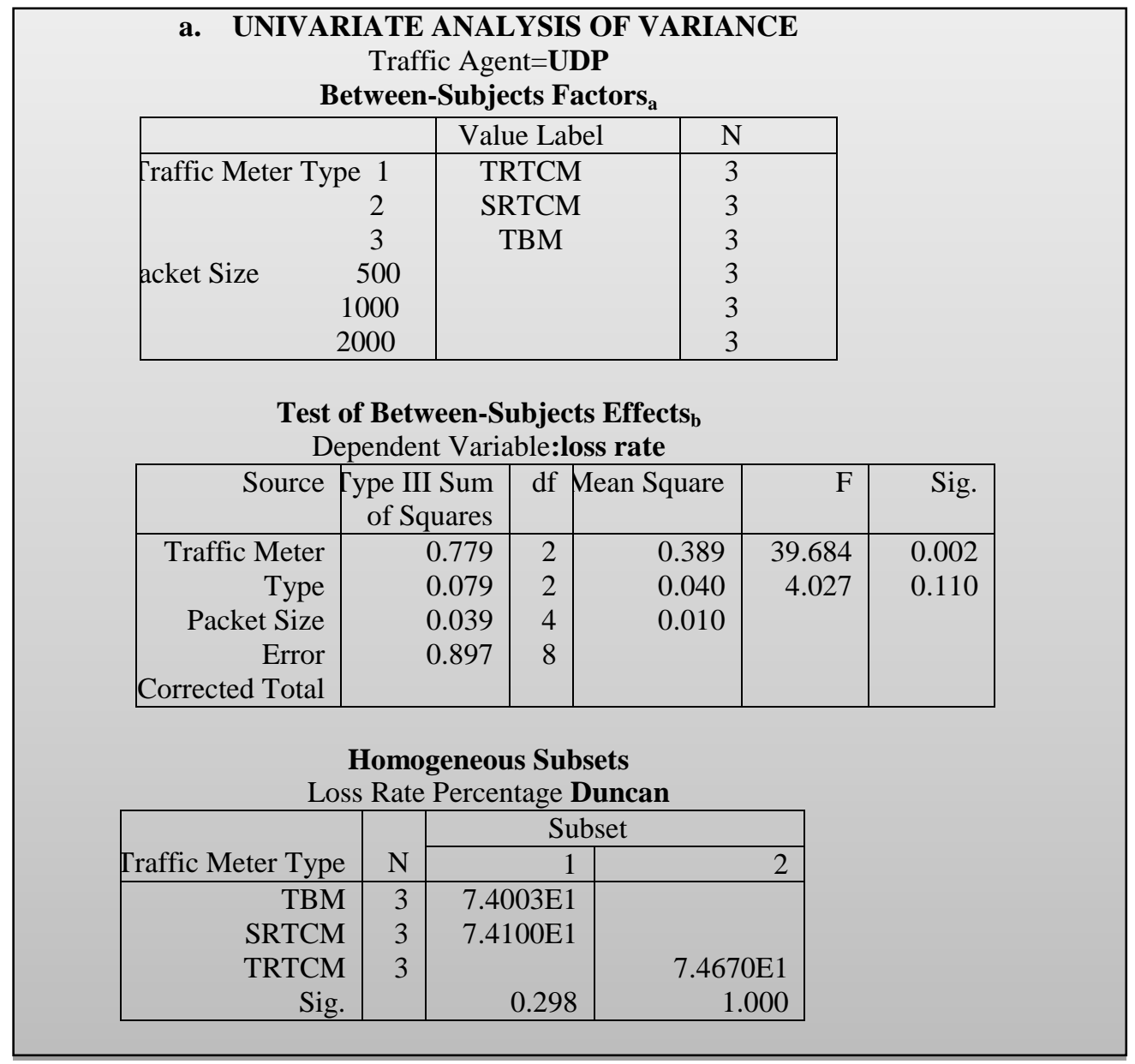

Figure 11: ANOVA analyses for loss rate using UDP as traffic agent

\section{d. Analysis Based on One-way Packet Delay}

The lower the one-way packet delay value, the better the performance of the traffic meter algorithm. One-way packet delay was calculated by subtracting packets arrival time $(a(n))$ from departure time $(\mathrm{d}(\mathrm{n}))$ as stated in (Mezger \& Petr, 1995):

\section{Packet delay $\rightarrow d(n)-a(n)$}

Comparing TRTCM, SRTCM and TBM in terms of one-way packet delay, Table 4 shows that TBM algorithm was ranked first, followed by SRTCM and then TRTCM. The results gotten for each traffic meter in Table 3 were represented graphically with bar chart in Figure 12. Figure 13 showed the 2-way ANOVA analyses with p-value of 0.006 which makes the null hypothesis to be rejected for alternative hypothesis. It shows that there is significant difference among the traffic meters. Applications that require low loss rate value on UDP traffic agent could make use of TBM algorithm 
Table 4: One-way packet delay analyses for the traffic meters

\begin{tabular}{|l|l|l|l|}
\hline \multirow{2}{*}{ SCENERIOS } & \multicolumn{3}{|c|}{ TRAFFIC METERS } \\
\cline { 2 - 4 } & $\begin{array}{l}\text { TRTCM- } \\
\text { UDP }\end{array}$ & SRTCM-UDP & TBM-UDP \\
\hline 1st (500Bytes) & 0.4419 & 0.2978 & 0.06564 \\
\hline 2nd (1000Bytes) & 0.86667 & 0.58865 & 0.10664 \\
\hline 3rd (2000Bytes) & 0.86717 & 0.58663 & 0.10685 \\
\hline $\begin{array}{l}\text { Avg.One-way packet } \\
\text { delay }\end{array}$ & $\mathbf{0 . 7 2 5 3}$ & $\mathbf{0 . 4 9 1}$ & $\mathbf{0 . 0 9 3}$ \\
\hline Ranks & $\mathbf{3}^{\text {rd }}$ & $\mathbf{2}^{\text {nd }}$ & $\mathbf{1}^{\text {st }}$ \\
\hline
\end{tabular}

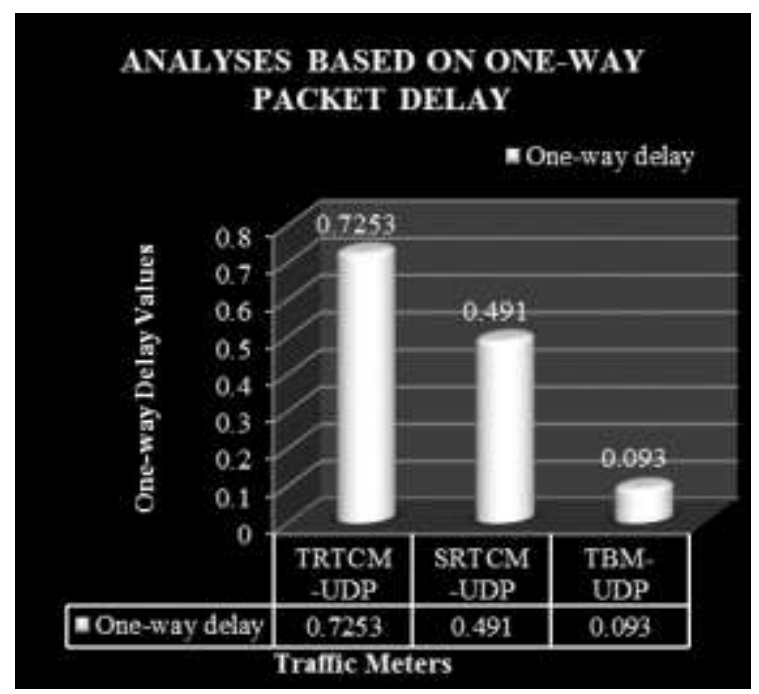

Figure 12: One-way packet delay analyses for the traffic meter algorithms

\section{Conclusion}

Looking at the four performance metrics that were used to evaluate the token bucket variants of traffic meter algorithms using UDP as traffic agent, TRTCM algorithm was ranked first for both throughput and fairness rate while TBM algorithm was ranked first for both loss rate and one-way packet delay. Applications that require high throughput and fairness rate could make use of TRTCM algorithm while the ones that require low loss rate and low one-way packet delay can make use of TBM algorithm for quality of service admission control in the internet as shown in Table 5 


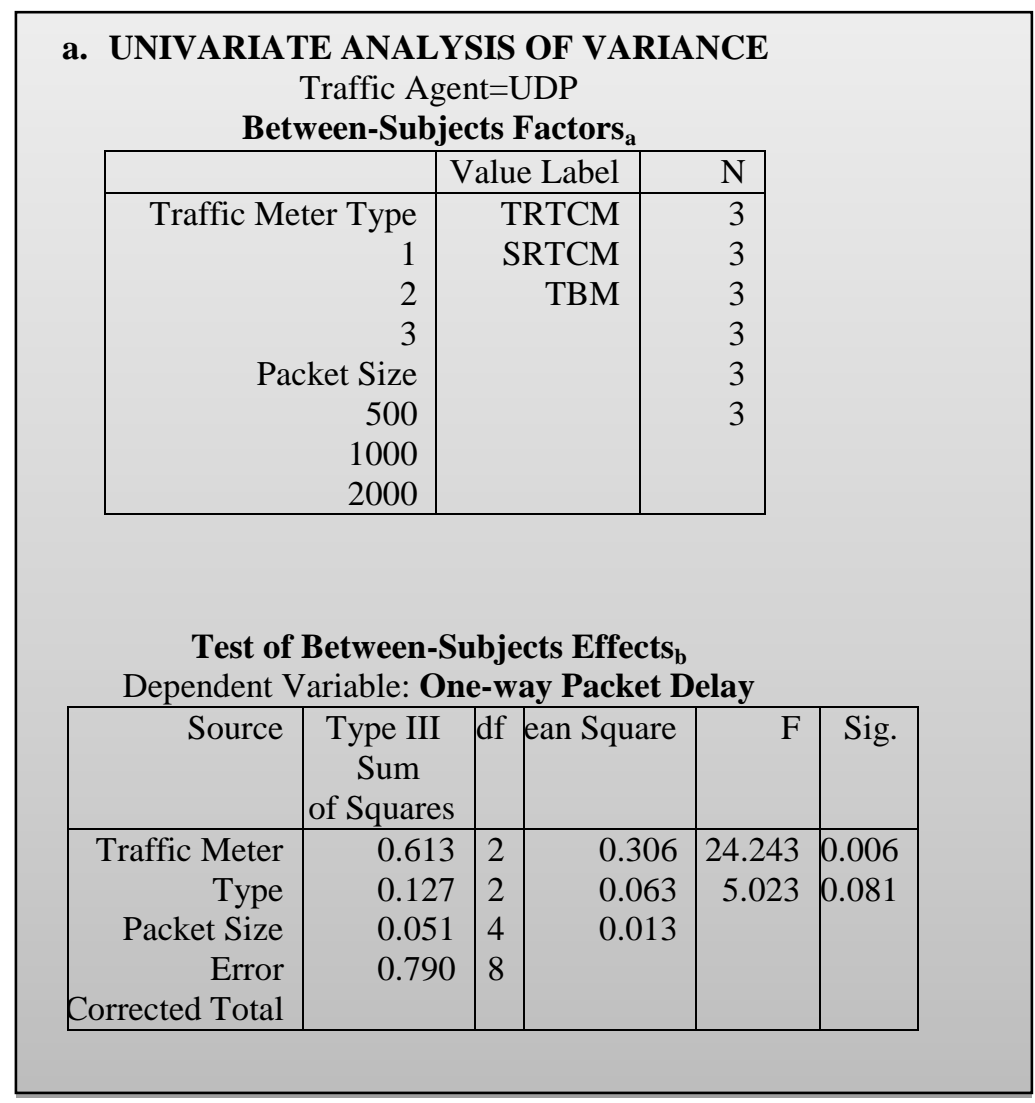

Homogeneous Subsets

One-way Packet Delay

Duncan

\begin{tabular}{|r|r|r|r|}
\hline & & \multicolumn{2}{|r|}{ Subset } \\
\cline { 3 - 4 } Traffic Meter Type & $\mathrm{N}$ & 1 & 2 \\
\hline TBM & 3 & 0.093043 & \\
SRTCM & 3 & & 0.491027 \\
TBM & 3 & & 0.725247 \\
Sig. & & 1.000 & 0.063 \\
\hline
\end{tabular}

Figure 13: ANOVA analyses for loss rate using UDP as traffic agent

Table 5: Ranking system analyses for meter algorithms using UDP as traffic agent

\begin{tabular}{|l|l|l|l|l|l|l|l|l|}
\hline \multirow{2}{*}{$\begin{array}{l}\text { TRAFFIC } \\
\text { METER }\end{array}$} & $\begin{array}{l}\text { PNE-WAY } \\
\text { PKT } \\
\text { DELAY }\end{array}$ & Rank & $\begin{array}{l}\text { FAIRNESS } \\
\text { RATE }\end{array}$ & Rank & $\begin{array}{l}\text { THROU } \\
\text { GHPUT }\end{array}$ & Rank & $\begin{array}{l}\text { LOSS } \\
\text { RATE }\end{array}$ & Rank \\
\hline TRTCM $_{\text {AVG }}$ & 0.7253 & $3^{\text {RD }}$ & $\mathbf{0 . 2 5 9}$ & $\mathbf{1}^{\text {ST }}$ & $\mathbf{6 7 1 1 7}$ & $\mathbf{1}^{\text {ST }}$ & 74.67 & $2^{\text {ND }}$ \\
\hline SRTCM $_{\text {AVG }}$ & 0.491 & $\mathbf{2}^{\text {ND }}$ & 0.1279 & $2^{\text {ND }}$ & 66949 & $2^{\text {ND }}$ & 74.1 & $3^{\text {RD }}$ \\
\hline $\mathrm{TBM}_{\text {AVG }}$ & $\mathbf{0 . 0 9 3}$ & $\mathbf{1}^{\text {ST }}$ & $\mathbf{0 . 1 2 6}$ & $3^{\text {RD }}$ & 66684 & $3^{\text {RD }}$ & $\mathbf{7 4}$ & $\mathbf{1}^{\text {ST }}$ \\
\hline
\end{tabular}


International Journal of Science and Management Studies (IJSMS)

Volume: 01 Issue: 04
November to December 2018
E-ISSN: 2581-5946

www.ijsmsjournal.org

\section{REFERENCES}

[1] Andreozzi, S. (2000). DiffServ Simulations using the Network Simulator: requirements, issues and solutions.

[2] Blake, S., Black, D., Carlson, M., Davies, E., Wang, Z., \& Weiss, W. (1998). An architecture for differentiated services.

[3] Braden, R., Clark, D., \& Shenker, S. (1994). Integrated services in the internet architecture: an overview: rfc 1633, June.

[4] Carlson, M., Weiss, W., Blake, S., Wang, Z., Black, D., \& Davies, E. (1998). An architecture for differentiated services: RFC 2475, December.

[5] Clark, D. D., \& Fang, W. (1998). Explicit allocation of best-effort packet delivery service. Networking, IEEE/ACM Transactions on, 6(4), 362-373.

[6] Freed, M., Amara, S., \& Borella, M. (2006). System and method for traffic shaping based on generalized congestion and flow control: Google Patents.

[7] Georgoulas, S. (2007). Admission control and bandwidth management in IP differentiated services networks. University of Surrey.

[8] Heinanen, J., \& Guérin, R. (1999). A two rate three color marker.

[9] Jain, R. (1990). Congestion control in computer networks: Issues and trends. IEEE Network, 4(3), 24-30.

[10] Kurose, J., \& Ross, K. (2000). Computer Networking, A Top-down Approach Featuring the Internet Book: Pearson Education, Limited.

[11] Lochin, E., \& Anelli, P. (2009). TCP throughput guarantee in the DiffServ Assured Forwarding service: what about the results? annals of telecommunications-annales des télécommunications, 64(3-4), 215-224.

[12] Mezger, K., \& Petr, D. W. (1995). Trace Traffic Integration into Model-Driven Simulations.

[13] Miller, P. (2009). TCP/IP: The Ultimate Protocol Guide (Vol. 2): Universal-Publishers.

[14] Nordström, E. (2006). Course Compendium in Network Modeling: Citeseer.

[15] Oyetunji, M., Oladeji, F., \& Emuoyinbofarhe, O. (2012). Performance Evaluation of Traffic Meters: Token Bucket Marker and Two Rate Three Color Marker (trTCM) QoS Admission Control. International Journal of Computer Applications, 56(8).

[16] Pandit, K. (2006). Quality of service performance analysis based on network calculus. TU Darmstadt.

[17] Strauss, T., Kourie, D. G., \& Olivier, M. S. (2005). A simulation study of traffic conditioner performance. Paper presented at the Proceedings of the 2005 annual research conference of the South African institute of computer scientists and information technologists on IT research in developing countries. 